\title{
Crystal structure of 2,2-diphenyl-4-dimethylaminopentanenitrile, $\mathrm{C}_{19} \mathrm{H}_{22} \mathrm{~N}_{2}$
}

\author{
A. Amani*, G. Rezanejade Bardajee, F. Jafarpour, M. O. Razaghi, M. Yousefi Behzadi and A. Haeri
}

Pasteur Institute of Iran, Medicinal Chemistry, Building 69, 12 Farvardin - Enghelab, 13164 Tehran, Iran

Received August 26, 2005, accepted and available on-line October 24, 2005; CCDC no. 1267/1634
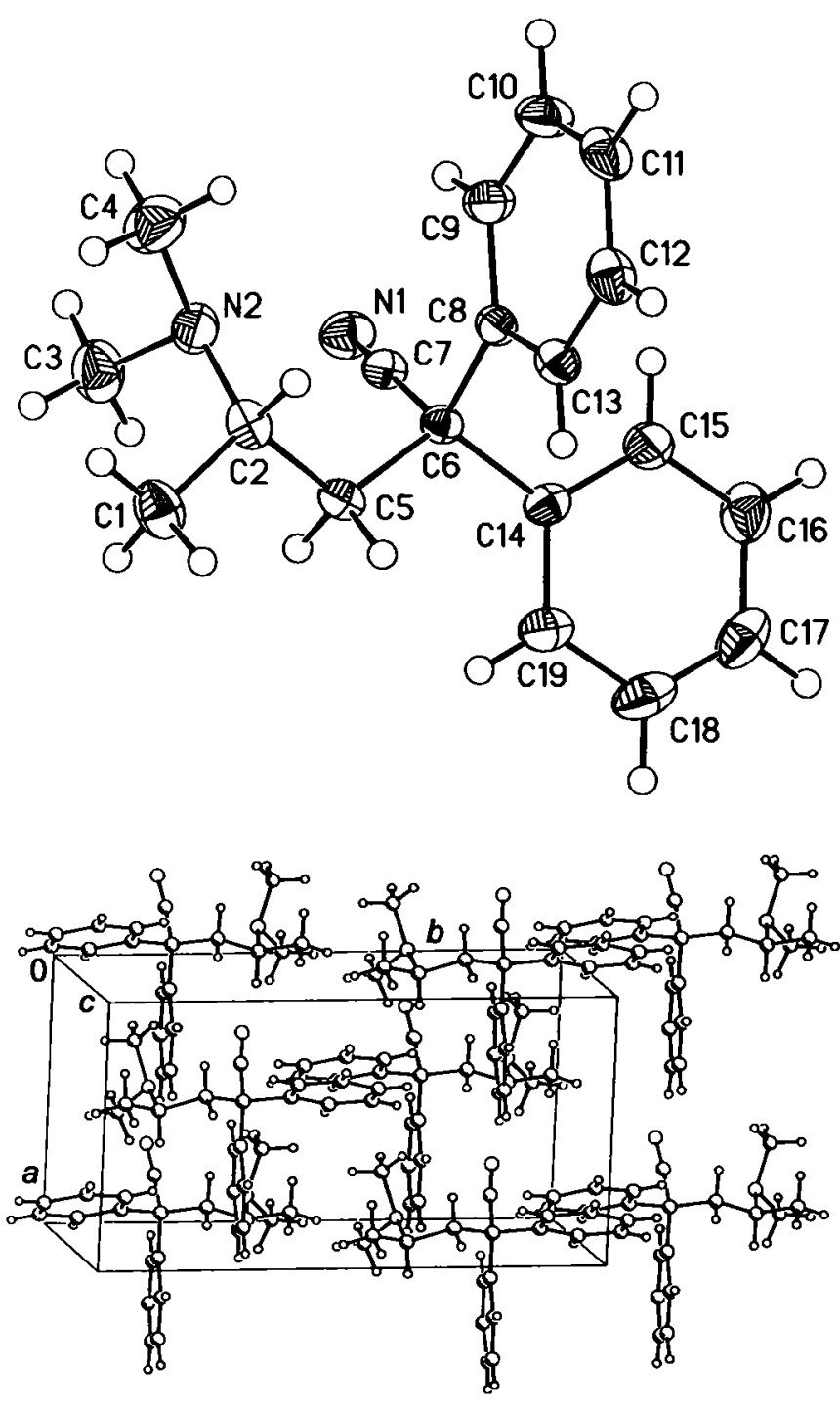

Abstract

$\mathrm{C}_{19} \mathrm{H}_{22} \mathrm{~N}_{2}$, monoclinic, $C 1 c 1$ (no. 9), $a=8.636(1) \AA$, $b=15.162(2) \AA, c=12.807(2) \AA, \beta=106.599(3)^{\circ}$,

$V=1607.1 \AA^{3}, Z=4, R_{\mathrm{gt}}(F)=0.044, w R_{\mathrm{ref}}\left(F^{2}\right)=0.088$,

$T=120 \mathrm{~K}$

\section{Source of material}

The title compound was synthesized by reaction of 1-dimethylamino-2-chloropropane hydrochloride and diphenylacetonitrile in the presence of $\mathrm{NaOH}$ as a base. 1-dimethylamino-2-chloro-

\footnotetext{
* Correspondence author (e-mail: amani121aa@yahoo.com)
}

propane was obtained from the reaction of thionyl chloride and 1dimethylamino-2-propanol whose preparation was described previously [1]. In a typical procedure, a $50 \% \mathrm{w} / v$ solution of sodium hydroxide in water $(12.5 \mathrm{ml}, 0.32 \mathrm{~mol})$ was added to a mechanically stirred suspension of diphenylacetonitrile $(15.0 \mathrm{~g}$, $0.08 \mathrm{~mol})$ and dibenzo-18-crown- $6(0.5 \mathrm{~g}$, cat.) in dimethylsulfoxide $(12.5 \mathrm{ml})$. The color rapidly deepened to an orange/brown. 1-Dimethylamino-2-chloropropane hydrochloride $(15 \mathrm{~g}, 0.095 \mathrm{~mol})$ was added in portions over $30 \mathrm{~min}$, this caused the temperature to rise to $30^{\circ} \mathrm{C}$. After the addition was completed the mixture was warmed to $45-50^{\circ} \mathrm{C}$ (water bath) and stirred for one hour. The reaction mixture was then allowed to cool to room temperature, poured into ice/water $(250 \mathrm{ml})$ and extracted with ethyl acetate $(3 \times 150 \mathrm{ml})$. The combined extracts were dried $\left(\mathrm{MgSO}_{4}\right)$, filtered and evaporated down to $100 \mathrm{ml}$. The product was extracted into $1 \mathrm{~N} \mathrm{HCl}(100 \mathrm{ml}+50 \mathrm{ml})$ and then again washed with ethyl acetate. The aqueous solution was basified with $2 \mathrm{M}$ sodium hydroxide and extracted into ethyl acetate $(3 \times 100 \mathrm{ml})$. The extracts were washed with saturated $\mathrm{NaCl}$ solution $(70 \mathrm{ml})$, dried $\left(\mathrm{MgSO}_{4}\right)$, and evaporated down to a yellow oil. This was chilled and titrated with cold hexane $(50 \mathrm{ml})$ to give the title compound (yield $39 \%$ ). Recrystallization from hexane gave crystals suitable for X-ray analysis (m.p. 91-92 ${ }^{\circ} \mathrm{C}$ ).

\section{Discussion}

Racemic methadone, 6-dimethylamino-4,4-diphenyl-3-heptanone, is used as a maintenance drug in the treatment of heroin addiction and severe pain symptoms. It also helps to combat the spread of HIV by reducing injection of heroin [2]. These and other unique properties of methadone led us to synthesize the methadone. In a multi-step preparation of methadone, the synthesis of 2,2-diphenyl-4-dimethyl aminopentanenitrile is a very important step in which we gained a series of crystals.

The crystal structure of the title compound is built up by only the $\mathrm{C}_{19} \mathrm{H}_{22} \mathrm{~N}_{2}$ molecules within which all bond lengths are almost in normal ranges (figure, top). $\mathrm{C6}$ atom is substituted with two phenyl groups and the $\mathrm{C} 8-\mathrm{C} 6-\mathrm{C} 14$ angle is $108.2(4)^{\circ}$. The $\mathrm{C} 7-\mathrm{C} 6-\mathrm{C} 14$ angle of $104.3(2)^{\circ}$ and $\mathrm{C} 2-\mathrm{C} 1-\mathrm{C} 16$ angle of $110.8(2)^{\circ}$ deviate slightly from the ideal value of $109.5^{\circ}$. The other bond angle around $\mathrm{C} 6$ are $\mathrm{C5}-\mathrm{C} 6-\mathrm{C7}\left(108.6(2)^{\circ}\right), \mathrm{C} 5-\mathrm{C} 6-\mathrm{C} 8\left(111.0(2)^{\circ}\right)$ and $\mathrm{C} 5-\mathrm{C} 6-\mathrm{C} 14\left(112.8(2)^{\circ}\right)$, which shows small steric strains between the substituents. The cyanide group twists very slightly out of the line of $\mathrm{C} 1-\mathrm{C} 2$ as seen by the $\mathrm{C} 6-\mathrm{C} 7-\mathrm{N} 1$ angle of $173.4(2)^{\circ}$. The planes of the two phenyl rings on $\mathrm{C} 6$ are oriented differently to each other with one in a horizontal and the other in a vertical manner. The arrangement of two molecules in the unit cell is in a fashion that intermolecular $\pi-\pi$ stacking interactions between two horizontal phenyl groups are achieved. These interactions were not observed for the vertical phenyl groups (figure, bottom). 
Table 1. Data collection and handling.

\begin{tabular}{ll}
\hline Crystal: & $\begin{array}{l}\text { colorless prism, } \\
\text { size } 0.6 \times 0.4 \times 0.4 \mathrm{~mm}\end{array}$ \\
Wavelength: & Mo $K_{a}$ radiation $(0.71073 \AA)$ \\
$\mu:$ & $0.67 \mathrm{~cm}^{-1}$ \\
Diffractometer, scan mode: & Bruker SMART $1000 \mathrm{CCD}, \varphi / \omega$ \\
$2 \theta_{\max }:$ & $60.06^{\circ}$ \\
$N(h k l)_{\text {measured, }} N(h k l)_{\text {hnique }}:$ & 7566,2322 \\
Criterion for $I_{\text {obs, }} N(h k l)_{\mathrm{gt}}:$ & $I_{\text {obs }}>2 \sigma\left(I_{\text {obs }}\right), 2073$ \\
$N(\text { param })_{\text {refined: }}$ & 190 \\
Programs: & SHELXTL [3], SHELXL-97 [4] \\
& \\
\hline
\end{tabular}

Table 2. Atomic coordinates and displacement parameters (in $\AA^{2}$ ).

\begin{tabular}{llllll}
\hline Atom & Site & $x$ & $y$ & $z$ & $U_{\text {iso }}$ \\
\hline H(1A) & $4 a$ & 0.6016 & 0.5007 & 0.1346 & 0.055 \\
H(1B) & $4 a$ & 0.5759 & 0.4373 & 0.0345 & 0.055 \\
H(1C) & $4 a$ & 0.7485 & 0.4487 & 0.1162 & 0.055
\end{tabular}

Table 2. Continued.

\begin{tabular}{llllll}
\hline Atom & Site & $x$ & $y$ & $z$ & $U_{\text {iso }}$ \\
\hline H(2A) & $4 a$ & 0.4838 & 0.3664 & 0.1631 & 0.033 \\
H(3A) & $4 a$ & 0.8705 & 0.4138 & 0.4096 & 0.063 \\
H(3B) & $4 a$ & 0.8437 & 0.4656 & 0.2999 & 0.063 \\
H(3C) & $4 a$ & 0.9019 & 0.3673 & 0.3083 & 0.063 \\
H(4A) & $4 a$ & 0.6130 & 0.4403 & 0.4203 & 0.070 \\
H(4B) & $4 a$ & 0.4599 & 0.4103 & 0.3280 & 0.070 \\
H(4C) & $4 a$ & 0.5580 & 0.4934 & 0.3108 & 0.070 \\
H(5A) & $4 a$ & 0.7689 & 0.2873 & 0.1448 & 0.029 \\
H(5B) & $4 a$ & 0.5977 & 0.2768 & 0.0615 & 0.029 \\
H(10A) & $4 a$ & 0.5099 & 0.2019 & 0.3779 & 0.035 \\
H(11A) & $4 a$ & 0.2459 & 0.1963 & 0.3895 & 0.044 \\
H(12A) & $4 a$ & 0.0309 & 0.1935 & 0.2333 & 0.043 \\
H(13A) & $4 a$ & 0.0778 & 0.1981 & 0.0643 & 0.039 \\
H(14A) & $4 a$ & 0.3403 & 0.2006 & 0.0515 & 0.031 \\
H(16A) & $4 a$ & 0.5707 & 0.0344 & 0.2447 & 0.038 \\
H(17A) & $4 a$ & 0.6073 & -0.0991 & 0.1665 & 0.047 \\
H(18A) & $4 a$ & 0.7066 & -0.1009 & 0.0159 & 0.048 \\
H(19A) & $4 a$ & 0.7716 & 0.0291 & -0.0533 & 0.047 \\
H(20A) & $4 a$ & 0.7365 & 0.1632 & 0.0245 & 0.037 \\
& & & & & \\
\hline
\end{tabular}

Table 3. Atomic coordinates and displacement parameters (in $\AA^{2}$ ).

\begin{tabular}{|c|c|c|c|c|c|c|c|c|c|c|}
\hline Atom & Site & $x$ & $y$ & $z$ & $U_{11}$ & $U_{22}$ & $U_{33}$ & $U_{12}$ & $U_{13}$ & $U_{23}$ \\
\hline $\mathbf{N}(1)$ & $4 a$ & $0.8505(2)$ & $0.1877(1)$ & $0.3866(2)$ & $0.030(1)$ & $0.039(1)$ & $0.0257(9)$ & $0.0018(8)$ & $0.0064(8)$ & $0.0001(8)$ \\
\hline$N(2)$ & $4 a$ & $0.6674(3)$ & $0.3796(1)$ & $0.2967(2)$ & $0.035(1)$ & $0.0275(9)$ & $0.0297(9)$ & $-0.0027(8)$ & $0.0080(8)$ & $-0.0022(8)$ \\
\hline$C(1)$ & $4 a$ & $0.6350(3)$ & $0.4463(2)$ & $0.1095(2)$ & $0.037(1)$ & $0.031(1)$ & $0.039(1)$ & $-0.004(1)$ & $0.007(1)$ & $0.009(1)$ \\
\hline$C(2)$ & $4 a$ & $0.6015(3)$ & $0.3699(2)$ & $0.1781(2)$ & $0.025(1)$ & $0.026(1)$ & $0.031(1)$ & $-0.0018(8)$ & $0.0071(9)$ & $0.0029(9)$ \\
\hline C(3) & $4 a$ & $0.8352(3)$ & $0.4091(2)$ & $0.3316(2)$ & $0.042(2)$ & $0.042(1)$ & $0.037(1)$ & $-0.011(1)$ & $0.002(1)$ & $0.001(1)$ \\
\hline C(4) & $4 a$ & $0.5658(4)$ & $0.4357(2)$ & $0.3429(3)$ & $0.059(2)$ & $0.038(1)$ & $0.046(2)$ & $0.007(1)$ & $0.021(1)$ & $-0.005(1)$ \\
\hline$C(5)$ & $4 a$ & $0.6544(3)$ & $0.2836(1)$ & $0.1382(2)$ & $0.0212(9)$ & $0.029(1)$ & $0.0224(9)$ & $-0.0009(8)$ & $0.0073(8)$ & $0.0033(8)$ \\
\hline$C(7)$ & $4 a$ & $0.7481(3)$ & $0.1966(1)$ & $0.3077(2)$ & $0.026(1)$ & $0.025(1)$ & $0.023(1)$ & $-0.0007(8)$ & $0.0102(8)$ & $0.0001(8)$ \\
\hline $\mathrm{C}(8)$ & $4 a$ & $0.6242(2)$ & $0.2000(1)$ & $0.1998(2)$ & $0.0181(9)$ & $0.0227(9)$ & $0.0204(8)$ & $0.0002(7)$ & $0.0064(7)$ & $0.0004(7)$ \\
\hline C(9) & $4 a$ & $0.4528(2)$ & $0.2007(1)$ & $0.2134(2)$ & $0.0221(9)$ & $0.0192(9)$ & $0.0251(9)$ & $0.0000(8)$ & $0.0099(8)$ & $0.0004(8)$ \\
\hline$C(10)$ & $4 a$ & $0.4238(3)$ & $0.2002(2)$ & $0.3147(2)$ & $0.031(1)$ & $0.033(1)$ & $0.027(1)$ & $0.0002(9)$ & $0.0116(9)$ & $-0.0012(9)$ \\
\hline C(11) & $4 a$ & $0.2648(3)$ & $0.1973(2)$ & $0.3216(2)$ & $0.039(1)$ & $0.040(1)$ & $0.041(1)$ & $0.001(1)$ & $0.026(1)$ & $-0.001(1)$ \\
\hline C(12) & $4 a$ & $0.1362(3)$ & $0.1959(2)$ & $0.2283(2)$ & $0.026(1)$ & $0.031(1)$ & $0.056(2)$ & $0.000(1)$ & $0.022(1)$ & $-0.001(1)$ \\
\hline C(13) & $4 a$ & $0.1643(3)$ & $0.1980(2)$ & $0.1273(2)$ & $0.023(1)$ & $0.030(1)$ & $0.043(1)$ & $0.0000(9)$ & $0.008(1)$ & $0.001(1)$ \\
\hline C(14) & $4 a$ & $0.3221(3)$ & $0.1999(1)$ & $0.1197(2)$ & $0.023(1)$ & $0.027(1)$ & $0.029(1)$ & $-0.0009(8)$ & $0.0083(8)$ & $0.0010(9)$ \\
\hline$C(15)$ & $4 a$ & $0.6494(2)$ & $0.1133(1)$ & $0.1429(2)$ & $0.0195(9)$ & $0.028(1)$ & $0.0216(9)$ & $0.0021(8)$ & $0.0050(7)$ & $-0.0027(8)$ \\
\hline$C(16)$ & $4 a$ & $0.6112(3)$ & $0.0337(2)$ & $0.1848(2)$ & $0.035(1)$ & $0.027(1)$ & $0.037(1)$ & $0.0013(9)$ & $0.017(1)$ & $-0.002(1)$ \\
\hline$C(17)$ & $4 a$ & $0.6328(3)$ & $-0.0466(2)$ & $0.1378(3)$ & $0.037(1)$ & $0.028(1)$ & $0.055(2)$ & $0.002(1)$ & $0.015(1)$ & $-0.004(1)$ \\
\hline$C(18)$ & $4 a$ & $0.6927(3)$ & $-0.0476(2)$ & $0.0479(2)$ & $0.031(1)$ & $0.041(2)$ & $0.045(2)$ & $0.005(1)$ & $0.005(1)$ & $-0.018(1)$ \\
\hline C(19) & $4 a$ & $0.7310(3)$ & $0.0301(2)$ & $0.0066(2)$ & $0.032(1)$ & $0.054(2)$ & $0.032(1)$ & $0.007(1)$ & $0.011(1)$ & $-0.012(1)$ \\
\hline C(20) & $4 a$ & $0.7099(3)$ & $0.1110(2)$ & $0.0534(2)$ & $0.029(1)$ & $0.040(1)$ & $0.026(1)$ & $0.002(1)$ & $0.0096(9)$ & $-0.002(1)$ \\
\hline
\end{tabular}

Acknowledgment. The support by the Pasteur Institute of Iran is gratefully acknowledged.

\section{References}

1. Barnett, C. J.: Modification of methadone synthesis process step. US Patent no. US4048211 (1977)

2. Hull, J. D.; Scheinmann, F.; Turner, N. J.: Synthesis of optically active methadones, LAAM and bufuralol by lipase-catalysed acylations. Tetrahedron: Asymm. 14 (2003) 567-576 and references cited therein.
3. Sheldrick, G. M.: SHELXTL. Structure Determination Software Suite. Version 5.10. Bruker AXS, Madison, Wisconsin, USA 1998.

4. Sheldrick, G. M.: SHELXI-97. Program for the Refinement of Crystal Structures. University of Göttingen, Germany 1997. 